\title{
Editorial
}

\section{The work of WHO in the Region: a review of 2014}

\author{
Ala Alwan ${ }^{7}$
}

This December issue of EMHJ presents me with the opportunity to look back on what has been a unique year in many ways. Readers may not be widely aware of the range of work that WHO undertakes in the Region and of the contributions made by its staff and experts.

We made good progress in 2014 in the five priority areas of work endorsed by our Member States: health systems strengthening, control of communicable diseases, maternal and child health, noncommunicable diseases and emergency preparedness and response. This resulted in some important and innovative products to support health development in the Region, including a framework for health information systems and core indicators, a framework for action on advancing universal health coverage, and an updated framework for action to implement the UN political declaration on prevention and control of noncommunicable diseases. WHO is supporting the nine high-burden countries as they move forward with implementing the plans to accelerate achievement of the Millennium Development Goals 4 and 5.

A successful session of the Regional Committee, which comprised representatives from the Member States, was concluded with the endorsement of just three resolutions focused on the strategic action needed in a few critical areas, including health security and noncommunicable diseases. We will now look to getting on with the work in hand and producing results in the areas we have promised. We have also made some progress in streamlining management structures, and in transparency, accountability and monitoring of our output. We will continue to pursue quality in our support to Member States.

2014 saw an unprecedented number of emergency events across several countries, in particular Iraq, Jordan, Libya, Pakistan, Palestine, Somalia, Syria and Yemen. Of the five high-level grade 3 emergencies to which WHO is responding around the world, two of them are in this region, in Syria and Iraq. In total, including the number of protracted crises in the Region, of the 22 Member States, about two thirds are facing or have faced major emergencies and crises in 2014. The situation is intense and the potential health threats and consequences will continue to be a concern to us all.

On visits this past year to Mogadishu, Gaza, Ramallah, Baghdad, Erbil and Damascus I witnessed the work being carried out by WHO staff and nationals in exceptionally demanding conditions. We have worked intensely, with national and United Nations colleagues and other partners, to respond to developments on the ground. Key public health measures in such situations have included situation assessment, ensuring safe drinking-water and sanitation, strengthening early warning systems for the detection of diseases, and pre-positioning medicines and medical supplies, in addition to emergency mass vaccination campaigns. However, there is clearly a need to strengthen our emergency response and reinforce our capacity in coordinating international and regional health relief activities in the crisis-affected countries.
It has been a year also that has reminded us, yet again, that infectious diseases, for all we may think we have them under control, can reappear at any time. We commenced the year with a major push to prevent further polio outbreaks and to find solutions that will finally enable polio eradication. Despite the setback in Pakistan, where progress is deliberately impeded by sustained violence, our response and the extensive campaigns conducted in Syria and neighbouring countries, as well as Somalia, were effective and appreciated by the international community. Mid-year, we put major effort into working with countries in the Region, in particular Saudi Arabia, to contain the novel coronavirus now known as Middle East respiratory syndrome (MERS). We have ended the year with helping Member States in strengthening preparedness to deal with Ebola, conducting assessment missions in almost all countries in the space of just two months. Ebola will continue to be a global concern for some months to come but I hope that these assessments will have impact and strengthen countries ability to protect against potential imported cases, and to deal with them if they occur.

Our experience in managing emergencies and the current global experience in managing the Ebola outbreak, demonstrate the extent to which the world, including our region, is ill prepared to respond to serious public health emergencies. In particular, public health capacity to detect, adjust and respond to emerging health threats still needs to be considerably strengthened. 
As we look forward to a new year, I anticipate that the circumstances will continue to be challenging. Clearly, countries in crisis expect WHO to do more in scaling up response action and technical support in the weeks and months to come. Our region currently hosts more than $50 \%$ of the world's refugee population. It is crucial that we further strengthen our work in order to meet the huge needs of the populations concerned, as well as lead the efforts for efficient recovery of the health sector. In Pakistan, the response of the government, $\mathrm{WHO}$ and all the partners in the coming months will be a key factor in completing the job of polio eradication.
Needless to say, 2015 will be a challenging year for WHO. The world looks to WHO for leadership, placing their confidence and trust in our ability to respond to their expectations. We recognize that success in meeting the challenge will depend on our technical support, dedication and performance. 